\section{SPLENIC FEVER IN THE ARGENTINE REPUBLIC 1}

THE author stated that he did not think any one who had worked much on the subject of splenic fever could doubt that the bacilli which caused that disease were capable of considerable variation in their effects on animals and man. Whether this disease, which is without doubt the one which has been most thoroughly investigated of all zymotic affections, gave any support to the views of Dr. Carpenter was another matter, but there could be no doubt that the Bacillus anthracis can be so modified by artificial means that the disease which it produces when introduced into animals, such as sheep and cattle, varies considerably as to duration, amount of fever produced, as well as to its mortality.

That, on the other hiand, this bacillu; has at least a very strong tendency to retain the characters which it at present presents in Europe is shown by the fact that in the Argentine Republic,--into which the affection was introduced at least thirty years ago, and where the conditions are very different from those which exist in Europe, - we find that the minute organism retains its characteristic form and the properties with which we are so well acquainted in Europe, and that the disease which it produces is practically identical with the European disease. That it should vary in some particulars is perhaps only to be expected, but Dr. Roy preferred leaving that point to be treated of elsewhere and uccupying the time at his disposal with an account of the observations which he had made as to the means of protecting from the disease by means of inoculation.

Some six months ago he had been requested by a City com pany who possess a large tract of land in the Argentine Republic, to pay a visit to their property in order to investigate a disease which was causing much mortality amongst the cattle, sheep, and horses, and which was affecting the employés as well to a very serious extent. This disease, he found on arriving at the River Plate, was splenic fever, of which the absence of efficient veterinary surgeons and the general apathy of the owners of stock had prevented the recognition. Having spent some time in studying the characters of the disease, he proceeded to make observations on the be t mean; of protecting the stock by means of inoculation, which work was much facilitated by the liberality of the company (the "I.as Cabezas Estancia Company") who gave him "carte blanche" as to the number of animals which he might envloy for his experiments. Having previously found, in a small series of observations made in this country in conjunction with Dr. E. Klein, that splenic fever virus from white mice was of the proper strength to protect sheep from the disease, he proceeded in the same lines, employing such animals as were available to produce the inoculating fluid. After a number of aniwals had been tried, he found that the blood of Biscachas (prairie dos) which had died of the disease gave satisfactory re-ults when used to inoculate cattle and horses. It was, however, a little two powerful, as I or 2 per cent. of the cattle so inoculated died. The pecuniary loss entailed by this was, however, more than counterbalanced by the arrest of the mortality from the natural affection. With regard to sheep, greater difficulties were encountered, and no animals were found giving a viras sufficiently mitigated to cause only a slight form of the disease with subsequent protection. Unfortunately it was impssible to repeat on a large scale the successful experiments which Dr. Roy and Dr. Klein had made with virus from white mice, these animals not being obtainable. Virus from field-mice and rats did not prove satisfactory.

Under these circumstances the speaker then proceeded to investigate the results obtained by artificial mitigation of the bacilli in the laboratory. He first employed the method of Toussaint, which consists in warming the fluid containing the pathogenic organisms to a temperature of $55^{\circ} \mathrm{C}$. for a period varying from a half hour to one hour and a half. It was found possible by this means to diminish the strength of the virus so that it took longer to kill, and by graduating the duration of the heating it is not difficult to obtain a virus which will only kill a sinall percentage of the animals inoculated. But unfortunately, in weakening the virulence of the organised poison, this process weakens also its power of protecting from a second attack, and it was easy to kill the animals so inoculated by subsequently introducing into their system strong virus which had not been sub jected to heat. This metbod having failed, Dr. Roy proceeded to Buenos Ayres, where, in the laboratory of the "Collegia

\footnotetext{
${ }^{3}$ Abstract of a paper read at the British Association by Dr. C. S. Roy.
}

Nacional," which was kindly placed at his disposal, he manipulated the virus by the method of Pasteur, which consisis in cultivating the virus in sterilised chicken broth at a temperature of $42^{\circ}-43^{\circ} \mathrm{C}$. At this temperature the bacilli grow much les readily than at one more nearly approaching blood heat. The hacill so cultivated diminish in virulence day by day, and after being cultivated for six or eight days no longer caused the death of full-grown sheep, although they still killed lambs and prairie dogs.

Careful experiments with inoculating fluid prepared in this manner showed that with it a slight fever could be induced which suffices to protect, at least for some time, from a second attack of the disease. The same objection, however, which characterises the inoculating fluid prepared by Toussaint's method exists, though to a less extent, with regard to Pasteur's fluid ; in the case of the latter as well as the first named, the protecting power is seriously diminished at the same time that the virulence of the bacilli (as indicated by the mortality) is le sened. With care it is possible, however, to prepare a liquid which, while its virulence has been brought sufficiently below the lethal limit to insure that none of the inoculated animals succumb to the inoculation, still retains enough protecting power to enable the sheep to resist the effects of strong virus employed some ten to fifteen days after the first inoculation. Dr. Roy was, therefore, able to confirm the assertions of $M$. Pasteur regarding the attributes of his inoculating fluid in so far that it is possible effectually to protect sheep from anthrax by its use. Still, it was impossible to overlook the fact that its employment necessitated very careful graduation of the strength of the mitigated virus to the resisting power of the animals inoculated. The speaker did not think that the method was one which was likely to be adopted univer. sally, and he rather looked forward to the general acceptation of some inoculating fluid which had been mitigated by cultivation in the bodies of some animal distinct in species from that which it was desired to protect. In the case of cattle the virus taken from Biscachas seemed to protecc in all cases, whether the illness produced by the inoculation was mild or severe. It was to be hoped that more extended inquiries would confirm the favourable results obtained by employing the virus from white mice to protect sheep. The speaker stated that he proposed communicating the results of his observations on this subject to the Royal Society at an early date.

\section{SUGGESTIONS FOR FACILITATING THE USE} OF $A$ DELICATE BALANCE

IN some experiments with which I have lately been occupied, a coil of insulated wire, traversed by an electric current, was suspended in the balance, and it was a matter of necessity to be able quickly to check the oscillations of the beam, so as to bring the coil into a standard position corresponding to the zero of the pointer. A very simple addition to the apparatus allowed this to be done. The current from a Leclanché cell is led into an auxiliary coil of wire coaxial with the other, and is controlled by a key. When the contact is made, a vertical force acts upon the suspended coil, but ceases as soon as the contact is broken. After a little practice, the beam may be brought to rest at zero at the first or second application of the retarding force.

This control over the oscillations has been found so convenient that $I$ have applied a similar contrivance in the case of ordinary weighings, and my object in the present note is to induce chemists and others experienced in such operation; to give it a trial. Two magnets of steel wire, three or four inches long, are attached vertically to the scale pans, and underneath one of them is fixed a coil of insulated wire of perhaps fifty or one hundred turns, and of four or five inches in diameter. The best place for the coil is immediately underneath the bottom of the balance case. It is then pretty near the lower pole of the magnet, and is yet out of the way. The circuit is completed tbrough a Leclanché cell and a common spring contact key, placed in any convenient position. The only precaution required is not to bring other magnets into the neighbourhood of the balance, or, at any rate, not to move them during a set of weighings.

The other point as to which I wish to make a suggestion relates to the time of vibration of the beam. I think that with the view of obtaining a high degree of sensitiveness the vibrations are often made too slow. Now the limit of accuracy depents more upon the smallness of the force which can be relied upon 